\title{
Conglomeración de precursores de tensión arterial elevada en adolescentes
}

\author{
Carlos M. Arango ${ }^{\mathrm{a}, \mathrm{b}, *}$, Lucía Lema ${ }^{\mathrm{b}}$, José A. Petro ${ }^{\mathrm{b}}$, Willinton J. Watts ${ }^{\mathrm{b}}$ y Diana C. Páez ${ }^{\mathrm{c}}$ \\ a Instituto Universitario de Educación Física, Universidad de Antioquia, Medellín, Colombia

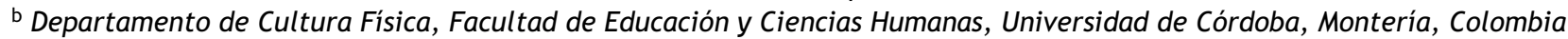 \\ c Departamento de Salud Pública, Universidad de los Andes, Bogotá, Colombia
}

Recibido el 11 de marzo de 2013; aceptado el 12 de mayo de 2014

Disponible en Internet el 6 de diciembre de 2014

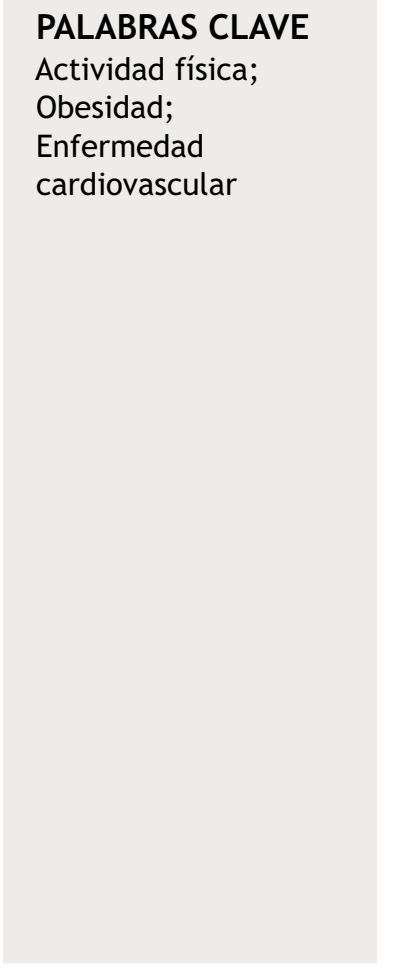

\begin{abstract}
Resumen
Introducción: La tensión arterial elevada y los precursores de enfermedad cardiovascular, pueden iniciarse en la infancia y mantenerse hasta la adultez. Estos precursores ocurren frecuentemente en conglomerado.

Objetivos: Analizar la conglomeración de precursores de tensión arterial alta en adolescentes y determinar la asociación de la frecuencia acumulada de estos con la tensión arterial elevada. Métodos: Estudio transversal en una muestra de 546 escolares de 11 a 18 años de la ciudad de Montería. Se tomaron medidas de tensión arterial, índice de masa corporal, perímetro abdominal, capacidad cardiorrespiratoria, niveles de actividad física y comportamientos sedentarios. Las asociaciones entre tensión arterial elevada y frecuencia acumulada de precursores fueron exploradas con modelos de regresión logística.

Resultados: La combinación de precursores más frecuente fue la presencia de dos precursores, inactividad física y tiempo excesivo de televisión (30,3\%). Le siguen la aparición simultánea de tres precursores, baja capacidad cardiorrespiratoria, inactividad física y tiempo excesivo de televisión (18,3\%). Aquellos adolescentes con dos o más precursores presentaron cifras de tensión arterial significativamente más altas en comparación con aquellos en quienes se encontró una acumulación menor de precursores. La tensión arterial elevada se asocia de manera significativa con la acumulación de precursores.

Conclusión: La conglomeración de dos o más precursores se asoció de manera significativa con la tensión arterial elevada, hallazgos que indican que se requieren intervenciones para reducir la presencia y acumulación de los precursores estudiados y así prevenir la adquisición de cifras de tensión arterial elevada en los adolescentes de Montería.

(c) 2013 Sociedad Colombiana de Cardiología y Cirugía Cardiovascular. Publicado por Elsevier España, S.L.U. Todos los derechos reservados.
\end{abstract}

\footnotetext{
* Autor para correspondencia.

Correo electrónico: cma31@students.uwf.edu (C.M. Arango).
} 


\section{KEYWORDS}

Physical activity; Obesity; Cardiovascular disease

\section{Conglomeration of high blood pressure precursors in adolescents}

\begin{abstract}
Introduction: High blood pressure and cardiovascular disease precursors may start in childhood and continue until adulthood. Usually these precursors occur in cluster.

Objectives: The objectives of the study were to analyze the cluster of high blood pressure precursors and to analyze the association of the cumulative frequency of these precursors with high blood pressure.

Methods: Cross-sectional study in a sample of 546 students aged 11 to 18 years in the city of Montería. Blood pressure, body mass index, waist circumference, cardiorespiratory fitness, physical activity and sedentary lifestyle were measured. The associations between high blood pressure and the cumulative frequency of precursors were explored with logistic regression models.

Results: The most frequently found combination of precursors was the presence of two precursors, physical inactivity and excessive TV time (30.3\%). It follows the simultaneous occurrence of three precursors: low cardiorespiratory fitness, physical inactivity, and excessive TV time (18.3\%). Those adolescents with two or more precursors showed a significantly higher occurrence of high blood pressure compared to those in whom a lower accumulation of precursors was found. The occurrence of high blood pressure is significantly associated with the accumulation of precursors.

Conclusion: The conglomeration of two or more precursors was significantly associated with the occurrence of high blood pressure. These findings indicate that interventions are needed to reduce the occurrence and accumulation of precursors studied, as well as to prevent the acquisition of high blood pressure in adolescents of Montería.

(c) 2013 Sociedad Colombiana de Cardiología y Cirugía Cardiovascular. Published by Elsevier España, S.L.U. All rights reserved.
\end{abstract}

\section{Introducción}

La tensión arterial elevada es uno de los principales contribuyentes a la carga mundial de morbimortalidad prematura $^{1}$, y es responsable de más de 7 millones de muertes cada año a nivel global ${ }^{2}$. Las estimaciones epidemiológicas más recientes indican que la tensión arterial elevada afecta al $26,3 \%$ de los hombres y al $19,7 \%$ de las mujeres en las Américas $^{3}$, al $29,1 \%$ de la población en Latinoamérica ${ }^{4}$, y al 34,3 y $26,5 \%$ de los hombres y de las mujeres en Colombia, respectivamente ${ }^{3}$. En Montería, Córdoba, ciudad situada en la costa caribe colombiana, la enfermedad cardiovascular ha sido la primera causa de muerte desde $1998^{5}$.

El proceso acelerado de urbanización que caracteriza a Latinoamérica se ha asociado con el incremento de enfermedades crónicas no transmisibles en la región ${ }^{6}$. Estos cambios urbanos han alterado, no solo el perfil epidemiológico de la población adulta, sino también de la población infantil. De hecho, en Colombia, y en un lapso de 5 años, el sobrepeso en niños de 10 a 17 años pasó del 13,7\% en 2005 al $16,7 \%$ en 2010, y el tiempo excesivo dedicado a ver televisión (más de 2 horas día) se incrementó de 34,4 a $38,8 \%{ }^{7}$.

Hay estudios que han evidenciado que la tensión arterial elevada y los precursores de la enfermedad cardiovascular, tales como inactividad física, comportamientos sedentarios, sobrepeso y obesidad, obesidad abdominal y baja capacidad cardiorrespiratoria, pueden iniciarse en la infancia y mantenerse hasta la adultez ${ }^{8,9}$, hasta provocar daños en los tejidos del sistema cardiovascular ${ }^{10,11}$.
Además, el comportamiento sedentario, como el tiempo excesivo dedicado a ver televisión ( $>2$ horas día), se incrementó en un 4,4\% del 2005 al 2010, en niños y adolescentes colombianos $^{7}$. En Montería, un estudio reportó que el $16 \%$ de los niños tienen sobrepeso o son obesos y solo el $24,7 \%$ cumple con las recomendaciones de actividad física (ejemplo: realizar 60 minutos de actividad física al menos 5 días a la semana $)^{12}$. Estos precursores pueden ocurrir en conjunto o en conglomerado y pueden desencadenar alteraciones metabólicas que provocan un aceleramiento de los procesos de enfermedad cardiovascular desde la infancia ${ }^{10,11,13}$.

En Colombia, los patrones de conglomeración de precursores de tensión arterial elevada en adolescentes, descritos previamente, no han sido analizados en detalle. Por tanto, la información que se desprende del estudio es relevante para la implementación de estrategias efectivas enfocadas a la prevención de enfermedades crónicas desde la infancia.

\section{Objetivos}

- Analizar la conglomeración de cinco precursores de tensión arterial elevada (sobrepeso, obesidad abdominal, capacidad cardiorrespiratoria baja, inactividad física y comportamientos sedentarios) en adolescentes.

- Determinar la asociación de la frecuencia acumulada de estos precursores con la tensión arterial elevada.

\section{Métodos}

Se trata de un análisis secundario de un estudio de corte transversal realizado en Montería, Córdoba, Colombia en 2008. Se recolectó información de población adolescente 
escolarizada, de colegios públicos o privados de la ciudad. El protocolo del estudio fue aprobado previamente por el Comité de Investigación de la Universidad de Córdoba y se obtuvo consentimiento informado firmado por los padres de los escolares incluidos en el estudio. Se incluyeron estudiantes que decidieron participar de manera voluntaria en el estudio, con autorización de sus padres, y se excluyeron aquellos con discapacidad física o mental que imposibilitara su participación.

\section{Población}

El procedimiento de muestreo se describe con detalle en una publicación anterior ${ }^{12}$. Brevemente, se tuvieron en cuenta los registros de la Secretaría Municipal de Educación, en los que se indica que la población escolar en 2007 fue de aproximadamente 103.629 estudiantes (74,2\% en la zona urbana), en 136 Instituciones Educativas (77,2\% localizadas en la zona urbana). El muestreo se realizó en tres etapas. Primero se hizo el muestreo de instituciones educativas en Montería, teniendo en cuenta el carácter (público, privado) y la localización, para así obtener como resultado 14 escuelas, con un registro de matrículas de 13.413 estudiantes. En la segunda etapa, y con base en este marco muestral, se calculó el tamaño de la muestra en 390 estudiantes. Finalmente, en la etapa tres se realizó el muestreo aleatorizado simple de los escolares en las edades requeridas (11-18 años), con base en los reportes de matrículas por institución, con fracciones de muestreo que permitieron completar la muestra requerida para cada grupo de edad y sexo, proporcional al número total de matrículas de cada escuela. Se aplicó un sobremuestreo con el fin de garantizar el número mínimo de sujetos para el estudio, de tal manera que se invitaron 578 estudiantes, de los cuales se obtuvieron datos completos de 546 . En total, la muestra quedó conformada por 546 escolares de 11 a 18 años de edad.

\section{Mediciones}

La recolección de información se llevó a cabo mediante la aplicación de los protocolos específicos para cada medición. La información fue recolectada por personal previamente capacitado. Los evaluadores fueron asignados a estaciones específicas, y por ende, cada uno de ellos midió las mismas variables durante toda la fase de recolección de información. Las valoraciones se aplicaron en el siguiente orden: tensión arterial, peso, talla, perímetro abdominal, cuestionarios y capacidad cardiorrespiratoria.

\section{Tensión arterial}

Se midió a través de la técnica de auscultación con un esfigmomanómetro (Welch Allyn), y un brazalete que se ajustara al tamaño del brazo del adolescente. Se siguió el protocolo del National High Blood Pressure Education Program - Working Group on High Blood Pressure in Children and Adolescents $^{14}$. Previo a la valoración, el adolescente estuvo en reposo, sentado durante al menos cinco minutos, con la espalda reclinada, los pies apoyados en el suelo y el brazo derecho apoyado, manteniendo la fosa cubital a la altura del corazón. Luego se midió la tensión arterial en tres ocasiones y se registró su promedio. Se definió tensión arterial elevada si los valores de tensión sistólica o diastólica fueron iguales o mayores al percentil 90 específico para sexo, edad y talla, o si la tensión arterial fue igual o superior a 120/80 $\mathrm{mmHg}^{14}$.

\section{Información antropométrica}

El peso se midió con una báscula Health o Meter con precisión de 200 gramos. El adolescente debía estar descalzo, en posición de pie, erguido, en buena ubicación sobre la báscula, mirada al frente y totalmente quieto. Se registró el peso en kilogramos. Para la talla se utilizó un tallímetro marca Seca, con medida en milímetros, localizado a 2 metros sobre una pared lisa. El adolescente debía estar descalzo y en posición erguida, con los brazos en posición anatómica; se procedió a colocar la base del tallímetro sobre la cabeza del participante mientras se le ordenó realizar una inspiración y sostener el aire por algunos segundos. Se registró la medida en metros y centímetros. El índice de masa corporal y sus percentiles se calcularon con base en las referencias de crecimiento de los Centros para el Control y Prevención de las Enfermedades. Fueron clasificados con sobrepeso si el percentil específico para sexo y edad de índice de masa corporal fue igual o superior a $85^{15}$. Se midió el perímetro abdominal con una cinta métrica inextensible sobre el tronco desnudo y en la línea media localizada entre la cresta ilíaca y la última costilla. Se les solicitó realizar una espiración para tomar la medida, la cual se registró en centímetros. La validez y reproducibilidad de esta medición ha sido documentada ${ }^{16,17}$. Se calculó el índice cintura-talla dividiendo el valor de la talla por el valor del perímetro. Los sujetos se clasificaron con obesidad abdominal si el valor del índice fue superior a $0,5^{18,19}$. En el análisis se incluyeron las variables de sobrepeso y obesidad abdominal como precursores independientes, puesto que los análisis de colinealidad así lo indicaron ( $\mathrm{VIF}=1,0)$.

\section{Capacidad cardiorrespiratoria}

Se valoró con el test de Leger (20 meter Shuttle run test, 20MST), diseñado para determinar la capacidad aeróbica máxima en niños, adolescentes y adultos saludables ${ }^{20}$. El test consiste en ir y volver entre dos líneas separadas por 20 metros, de acuerdo con el ritmo de una señal auditiva que indica el momento en que se debe tocar cada línea. La señal auditiva cambia cada minuto, de manera que el tiempo entre las señales es menor, lo que obliga al sujeto a incrementar la velocidad. La frecuencia de la señal inicial indica una velocidad de desplazamiento de $8,5 \mathrm{~km}^{*} \mathrm{~h}^{-1}$, y se incrementa cada minuto en $0,5 \mathrm{~km}^{*} \mathrm{~h}^{-1}$. El test finaliza cuando el sujeto no es capaz de seguir el ritmo de la señal para alcanzar cada línea. Se registra la máxima etapa alcanzada por el sujeto. Esta información se utiliza para predecir el consumo máximo de oxígeno $\left(\mathrm{VO}_{2 \max }\right)$, mediante la ecuación de Leger $^{20}$. La validez y reproducibilidad del test han sido documentadas ${ }^{21,22}$. Se utilizaron los estándares FITNESSGRAM específicos para sexo y edad ${ }^{23}$ con el fin de clasificar a los participantes en capacidad cardiorrespiratoria normal para aquellos que alcanzaron la zona saludable, y capacidad cardiorrespiratoria baja para quienes no lo hicieron. 
Actividad física y tiempo de televisión

Se les pidió a los estudiantes diligenciar un cuestionario de auto-reporte de actividad física y tiempo dedicado a ver televisión. Estas preguntas son parte de la Encuesta Mundial de Salud Escolar utilizada por la Organización Mundial de la Salud ${ }^{24}$. La actividad física se determinó con la pregunta: En los anteriores 7 días, ¿durante cuántos días participaste en actividad física por lo menos 60 minutos al día? (suma todo el tiempo que pasas en cualquier actividad física que aumente tu ritmo del corazón y que te haga respirar con dificultad parte del tiempo). Las opciones de respuesta fueron: 0 días, 1 día, 2 días, 3 días, 4 días, 5 días, 6 días y 7 días. Los estudiantes fueron categorizados como activos si reportaban 5 días o más de participación en actividad física; con las demás respuestas fueron categorizadas como inactivos. El tiempo de televisión se indagó con la pregunta: En un día normal de escuela, ¿cuántas horas ves la televisión? Las opciones de respuesta fueron: no veo televisión durante los días de escuela, menos de una hora diaria, una hora diaria, 2 horas diarias, 3 horas diarias, 4 horas diarias, 5 o más horas diarias. Las respuestas fueron categorizadas en dos grupos, menos de dos horas diarias y dos o más horas diarias (excesivo), de acuerdo con las recomendaciones en cuanto a tiempo dedicado a ver televisión en niños y adolescentes ${ }^{25}$.

\section{Covariables sociodemográficas}

Los estudiantes reportaron la edad y el género. El carácter de la escuela (pública o privada) fue usado como un proxy para posición socioeconómica.

\section{Análisis estadístico}

Se calcularon las prevalencias de cada uno de los precursores investigados, al igual que su frecuencia acumulada (acumulación de uno a 5 precursores). Se identificaron las diez combinaciones más frecuentes de precursores de tensión arterial. Las asociaciones entre tensión arterial elevada y tener 1, 2, 3, o 4 o más precursores fueron exploradas con modelos de regresión logística ajustados por sexo, localización de la escuela (urbana y rural) y carácter de la escuela (pública o privada). Las asociaciones entre la frecuencia acumulada de precursores y tensión arterial elevada se analizaron mediante el cálculo de razón de disparidad (OR, su sigla en inglés) con intervalos de confianza del 95\%. Todos los análisis se realizaron con el programa Stata, versión 12,0 y se utilizó el nivel de significación de 0,05. Debido a las características del muestreo, todos los análisis se llevaron a cabo teniendo en cuenta el efecto de los pesos muestrales y la conglomeración de la muestra, utilizando los comandos svy incluidos en el software Stata.

\section{Resultados}

Las características sociodemográficas, así como las prevalencias de tensión arterial elevada y de los precursores se resumen en la tabla 1 , de acuerdo con la frecuencia acumulada de conglomeración de precursores de tensión arterial elevada. Se identificó tensión arterial elevada en $21,7 \%$ de los adolescentes estudiados. Las prevalencias de los precursores de tensión arterial elevada fueron 14,9\% para sobrepeso, 14,5\% para obesidad abdominal, 41,9\% para capacidad cardiorrespiratoria baja, 77,3\% para inactividad

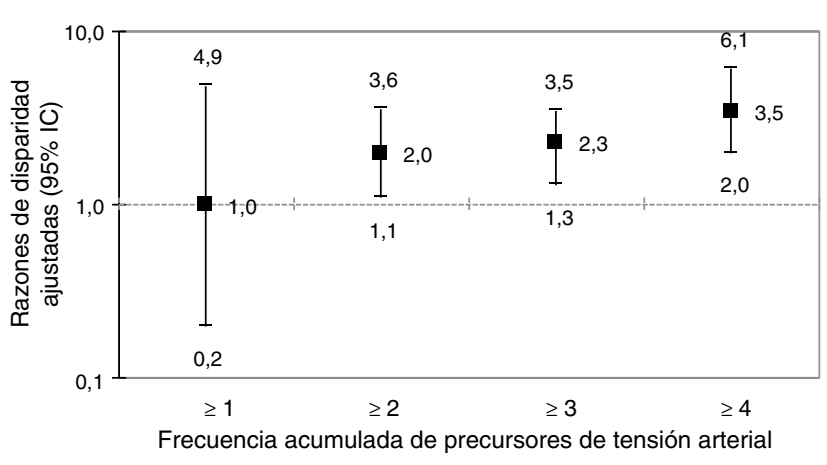

Figura 1 Asociaciones entre la frecuencia acumulada de precursores y tensión arterial elevada en 546 adolescentes de Montería, Colombia, 2008.*

* Las razones de disparidad fueron calculadas en modelos de regresión logística ajustados por sexo, localización de la escuela (urbana y rural) y carácter de la escuela (pública o privada).

física y $80,7 \%$ para tiempo de televisión excesivo (dos o más horas diarias). Los adolescentes con sobrepeso y obesidad abdominal tuvieron la más alta proporción de presencia de los cinco precursores estudiados. El 79\% de los adolescentes tuvieron 2 o más precursores de tensión arterial elevada.

Las primeras diez combinaciones más frecuentes de precursores de tensión arterial elevada se ilustran en la tabla 2. La combinación más común fue la presencia de dos precursores, inactividad física y excesivo tiempo de televisión $(30,3 \%)$ con ausencia de los demás precursores. Le siguieron la presencia de tres precursores, la baja capacidad cardiorrespiratoria, la inactividad física y el tiempo de televisión excesivo $(18,3 \%)$, más la presencia de un solo precursor, tiempo de televisión excesivo con la ausencia de los demás precursores $(9,2 \%)$.

Los resultados de la regresión logística se muestran en la figura 1. Las razones de disparidad ajustadas por sexo, localización de la escuela (urbana y rural) y carácter de la escuela (pública o privada) indican que la presencia de tensión arterial elevada se asocia de manera significativa con la acumulación de precursores de tensión arterial elevada en adolescentes, la cual fue significativamente más alta en aquellos con dos precursores que en aquellos que tuvieron menos de dos precursores (aOR: 2,0; 95\% IC: 3,6-1,1); igualmente, en aquellos con tres o más precursores (aOR: 2,3; $95 \%$ IC: $3,5-1,3)$ y en adolescentes con cuatro o más precursores (aOR: 3,5; 95\% IC: 6,1-2,0).

\section{Discusión}

En este estudio se analizó la conglomeración de cinco precursores de tensión arterial elevada, así como también la asociación de la frecuencia acumulada de estos con la tensión arterial elevada. Se detectó una prevalencia alta de tensión arterial elevada $(21,7 \%)$ en la muestra de adolescentes investigados, hallazgo que podría estar relacionado con la tasa elevada de mortalidad cardiovascular reportada en la región ${ }^{5}$. La prevalencia de tensión arterial elevada encontrada en este estudio es mayor en comparación con la más reciente prevalencia documentada en adolescentes estadounidenses de 8 a 17 años de edad (hombres 19,2\%, y mujeres $12,6 \%)^{26}$. También es mayor comparada con la 
Tabla 1 Características sociodemográficas de acuerdo con la frecuencia acumulada de factores de precursores de tensión arterial elevada en 546 adolescentes de Montería, Colombia, 2008.

\begin{tabular}{|c|c|c|c|c|c|c|}
\hline \multirow[t]{2}{*}{ Variables } & \multirow[b]{2}{*}{$\begin{array}{l}\text { Todos }(\%) \\
(n=546)\end{array}$} & \multicolumn{5}{|c|}{ Frecuencia acumulada de precursores de tensión arterial elevada } \\
\hline & & $\begin{array}{l}1 \%) \\
(n=535)\end{array}$ & $\begin{array}{l}2(\%) \\
(n=433)\end{array}$ & $\begin{array}{l}3(\%) \\
(n=192)\end{array}$ & $\begin{array}{l}4(\%) \\
(n=59)\end{array}$ & $\begin{array}{l}5(\%) \\
(n=13) \\
\end{array}$ \\
\hline \multicolumn{7}{|l|}{ Género } \\
\hline Hombre & 51,3 & 50,5 & 53,2 & 62,3 & 55,0 & 45,7 \\
\hline Mujer & 48,7 & 47,8 & 46,8 & 37,7 & 45,0 & 54,3 \\
\hline \multicolumn{7}{|c|}{ Localización de la escuela } \\
\hline Urbana & 77,4 & 77,6 & 77,4 & 78,9 & 82,0 & 85,8 \\
\hline Rural & 22,6 & 22,4 & 22,6 & 21,1 & 18,0 & 14,2 \\
\hline \multicolumn{7}{|c|}{ Carácter de la escuela } \\
\hline Público & 92,2 & 92,4 & 93,5 & 94,1 & 95,1 & 93,1 \\
\hline Privado & 7,8 & 7,6 & 6,5 & 5,9 & 4,9 & 6,9 \\
\hline \multicolumn{7}{|c|}{ Tensión arterial } \\
\hline Normal & 78,2 & 78,2 & 76,4 & 70,4 & 55,8 & 53,5 \\
\hline Elevada & 21,7 & 21,8 & 23,6 & 29,6 & 44,2 & 46,5 \\
\hline \multicolumn{7}{|l|}{ Peso corporal } \\
\hline Normal & 85,1 & 84,8 & 81,6 & 65,3 & 31,2 & 0,0 \\
\hline Sobrepeso & 14,9 & 15,2 & 18,4 & 34,7 & 68,8 & 100 \\
\hline \multicolumn{7}{|c|}{ Obesidad abdominal } \\
\hline No & 85,5 & 85,2 & 82,4 & 62,6 & 14,7 & 0,0 \\
\hline Sí & 14,5 & 14,8 & 17,5 & 37,4 & 85,3 & 100 \\
\hline \multicolumn{7}{|c|}{ Capacidad cardiorrespiratoria } \\
\hline Normal & 58,1 & 57,3 & 49,4 & 19,5 & 22,0 & 0,0 \\
\hline Baja & 41,9 & 42,7 & 50,6 & 80,5 & 78,0 & 100 \\
\hline \multicolumn{7}{|c|}{ Actividad física } \\
\hline Activo & 22,7 & 21,4 & 12,3 & 6,7 & 2,9 & 0,0 \\
\hline Inactivo & 77,3 & 78,6 & 87,7 & 93,3 & 97,1 & 100 \\
\hline \multicolumn{7}{|c|}{ Tiempo de televisión } \\
\hline Normal & 19,3 & 17,9 & 11,0 & 6,7 & 5,7 & 0,0 \\
\hline Excesivo & 80,7 & 82,1 & 89,0 & 93,3 & 94,3 & 100 \\
\hline
\end{tabular}

Los datos en negrita indican diferencias estadísticamente significativas $(p<0,05)$ entre el par de categorías comparadas y calculadas con la prueba chi-cuadrado.

* Las estimaciones están ponderadas teniendo en cuenta el diseño muestral. Todos los datos se muestran en porcentajes.

Tabla 2 Primeras diez combinaciones más frecuentes de precursores de tensión arterial elevada en 546 adolescentes de Montería, Colombia, 2008

\begin{tabular}{lllllll}
\hline $\begin{array}{l}\text { Número de } \\
\text { precursores }\end{array}$ & Sobrepeso & $\begin{array}{l}\text { Obesidad } \\
\text { abdominal }\end{array}$ & $\begin{array}{l}\text { Baja } \\
\text { CCR }\end{array}$ & $\begin{array}{l}\text { Inactividad } \\
\text { física }\end{array}$ & $\begin{array}{l}\text { Excesivo } \\
\text { tiempo de TV }\end{array}$ & Prevalencia (IC 95\%) \\
\hline 1 & - & - & - & - & + & $9,2(5,9-12,6)$ \\
& - & - & - & + & - & $6,9(2,5-11,2)$ \\
2 & - & - & - & + & + & $30,3(25,0-35,7)$ \\
& - & - & + & - & + & $5,7(3,7-7,8)$ \\
3 & - & - & + & + & + & $5,4(3,5-7,3)$ \\
4 & - & + & + & + & + & $3,6(15,3-21,3)$ \\
& + & + & - & + & + & $2,5(0,1-5,0)$ \\
5 & + & - & + & + & + & $1,7(0,5-2,9)$ \\
\hline
\end{tabular}

CCR: capacidad cardiorrespiratoria; IC: intervalo de confianza; TV: televisión; (-): ausencia del precursor; (+): presencia del precursor. 
prevalencia de tensión arterial elevada encontrada en un estudio realizado en Medellín en escolares de 6 a 18 años (tensión arterial sistólica elevada 1,3\%, y tensión arterial diastólica elevada $3,9 \%)^{27}$. Sin embargo, es similar a la prevalencia de $20,7 \%$ encontrada en adolescentes de 12 a 15 años de León, México ${ }^{28}$.

El estudio permitió detectar la prevalencia de los precursores de tensión arterial en los adolescentes. El tiempo excesivo de televisión (dos o más horas diarias) fue el precursor con la mayor prevalencia $(80,7 \%)$. Una cifra superior al $58,5 \%$ de prevalencia encontrada en población colombiana de edades entre 5 y 12 años $^{29}$. El tiempo excesivo sentado es un comportamiento que afecta la salud de forma negativa, independientemente del nivel de actividad física. De hecho, la evidencia epidemiológica sugiere una asociación entre el tiempo sentado, independientemente de la actividad física y el desarrollo de síndrome metabólico, diabetes tipo 2, obesidad $^{30}$, enfermedad cardiovascular ${ }^{30}$ y mortalidad por todas las causas ${ }^{31}$. También se ha reportado que los niños que permanecen por tiempo prolongado frente a la televisión están más expuestos a publicidad de alimentos, con bajos aportes nutricionales y altos contenidos calóricos, que junto con una alteración del balance energético corporal (mayor consumo y menor gasto calórico), los ponen en riesgo de sobrepeso y obesidad ${ }^{32,33}$. Otro factor importante respecto a la exposición a la televisión en niños y adolescentes, es la alteración en los patrones del sueño ${ }^{34}$, lo cual se asocia con mayores niveles de sobrepeso y obesidad ${ }^{35,36}$. La segunda prevalencia más alta fue la inactividad física (77,3\%), seguida por la capacidad cardiorrespiratoria baja $(41,9 \%)$, el sobrepeso $(14,9 \%)$ y finalmente, la obesidad abdominal (14,5\%). La prevalencia de sobrepeso es cercana al $16,7 \%$ de aquella encontrada en la Encuesta Nacional de Situación Nutricional (ENSIN, 2010) en niños de 10 a 17 años de edad ${ }^{7}$.

Igualmente, los resultados revelaron una conglomeración alta de precursores de tensión arterial elevada en la muestra. La combinación más frecuente fue inactividad con tiempo excesivo de televisión $(30,3 \%)$, seguida por la combinación de tres precursores: inactividad física, tiempo excesivo de televisión y capacidad cardiorrespiratoria baja $(18,3 \%)$. Estas conglomeraciones pueden explicarse teniendo en cuenta que precisamente el tiempo de televisión sustrae tiempo para la realización de actividades físicas, lo que conduce a una condición cardiorrespiratoria deficiente ${ }^{11}$. En la literatura se documenta la asociación de la tensión arterial elevada con estos precursores de manera independiente, por ejemplo con niveles bajos de actividad física ${ }^{37}$ o de capacidad cardiorrespiratoria ${ }^{38}$, obesidad abdominal ${ }^{39}$ y sobrepeso ${ }^{40}$.

Los análisis multivariados evidencian que la presencia de 2 o más precursores está asociada de modo significativo con la aparición de tensión arterial elevada. Cabe destacar que el $79 \%$ de los adolescentes estudiados presentaron 2 o más precursores, por tanto, estos hallazgos tienen relevancia para la salud pública si se tiene en cuenta que la enfermedad cardiovascular posee alta prevalencia en la población colombiana, ya que constituye la primera causa de morbimortalidad. Esto representa una carga económica para la salud del país debido al incremento de la demanda en la atención médica y en relación con la disminución de la capacidad productiva y la calidad de vida de los individuos ${ }^{41}$. En consecuencia, los hallazgos indican que es preciso formular intervenciones en educación para la salud, que tengan suficiente dimensión para prevenir la aparición individual y simultánea de estos precursores. Los resultados de este estudio sugieren que estas intervenciones deberían enfocarse en los tres precursores más prevalentes (tiempo excesivo de televisión, inactividad física y baja capacidad cardiorrespiratoria) con la intención de reducir tanto la conglomeración de precursores, como la aparición de tensión arterial elevada, y potencialmente otras enfermedades cardiovasculares y metabólicas. Igualmente se evidencia el efecto multiplicativo de tener 2, 3 o 4 o más precursores sobre la aparición de tensión arterial elevada en esta población. Así mismo, los resultados indican que existe una interacción biológica importante entre estos precursores de tensión arterial elevada. Sin embargo, se requieren más estudios con diseños longitudinales o experimentales que permitan revelar los mecanismos de interacción entre estos precursores y la elevación de la tensión arterial.

Los hallazgos documentados deben analizarse a la luz de las limitaciones del estudio. En primer lugar, la información referente al tiempo de ver televisión y actividad física fue auto-reportada, de allí que es posible que los datos hayan presentado sesgos de memoria. Pese a ello, las posibilidades de este tipo de sesgo están reducidas debido a que las preguntas están enmarcadas en una corta dimensión temporal. Segundo, el diseño transversal del estudio no permite hacer inferencia causal alguna. Por último, la clasificación de la tensión arterial se realizó con base en datos de población estadounidense, y ello pudo haber conducido a errores de clasificación. De otra parte, el estudio tiene fortalezas. De acuerdo con la información disponible, este es uno de los pocos estudios que explora la asociación entre la frecuencia acumulada de precursores y tensión arterial elevada en adolescentes de Latinoamérica. Adicionalmente, el diseño muestral permitió garantizar la representatividad de la muestra en cuanto a edad y sexo de los adolescentes de Montería.

En conclusión, se encontró una conglomeración de precursores de tensión arterial elevada en los adolescentes estudiados. La conglomeración de dos o más precursores fue alta y se asoció de manera significativa con la aparición de tensión arterial elevada, hallazgos que indican que se requieren intervenciones comportamentales para reducir la incidencia de los precursores estudiados y así prevenir la adquisición de tensión arterial elevada en los adolescentes de Montería.

\section{Financiación}

El estudio fue realizado sin financiación externa.

\section{Conflicto de intereses}

Los autores declaran no tener ningún conflicto de intereses.

\section{Agradecimientos}

A los estudiantes y rectores de las instituciones educativas que hicieron parte del estudio, así como a los estudiantes 
y profesores del programa de Licenciatura en Educación Física, Recreación y Deportes de la Universidad de Córdoba.

\section{Bibliografía}

1. Vasan RS, Larson MG, Leip EP, Evans JC, O’Donnell CJ, Kannel WB, et al. Impact of high-normal blood pressure on the risk of cardiovascular disease. N Engl J Med. 2001;345(18):1291-7.

2. Ezzati M, Lopez AD, Rodgers A, Vander Hoorn S, Murray CJ. Selected major risk factors and global and regional burden of disease. Lancet. 2002;360(9343):1347-60.

3. World Health Organization. World health statistics 2012. Geneva: WHO Press; 2012.

4. Lanas F, Avezum A, Bautista LE, Diaz R, Luna M, Islam S, et al. Risk factors for acute myocardial infarction in Latin America: the INTERHEART Latin American study. Circulation. 2007;115(9):1067-74.

5. Departamento Nacional de Estadística. Diez primeras causas de defunción, según departamento de residencia. 2009. 2012 [consultado 4 Abr 2012]. Disponible en: http://www.dane.gov.co/ index. php?option=com_content\&view=article\&id=788\&ltemid= 119

6. Barreto SM, Miranda JJ, Figueroa JP, Schmidt MI, Munoz S, Kuri-Morales PP, et al. Epidemiology in Latin America and the Caribbean: current situation and challenges. Int J Epidemiol. 2012;41(2):557-71

7. Instituto Colombiano de Bienestar Familiar y Profamilia. Encuesta Nacional de la Situación Nutricional en Colombia 2010 - ENSIN 2011 [consultado 12 Dic 2013]. Disponible en: http://www.bogotamasactiva.gov.co/files/Resumen \%20Ejecutivo\%20ENSIN\%202010.pdf

8. Sun SS, Grave GD, Siervogel RM, Pickoff AA, Arslanian SS, Daniels SR. Systolic blood pressure in childhood predicts hypertension and metabolic syndrome later in life. Pediatrics. 2007;119(2):237-46.

9. Bao W, Threefoot SA, Srinivasan SR, Berenson GS. Essential hypertension predicted by tracking of elevated blood pressure from childhood to adulthood: the Bogalusa Heart Study. Am J Hypertens. 1995;8(7):657-65.

10. Raitakari OT, Juonala M, Kahonen M, Taittonen L, Laitinen T, Maki-Torkko N, et al. Cardiovascular risk factors in childhood and carotid artery intima-media thickness in adulthood: the Cardiovascular Risk in Young Finns Study. JAMA. 2003;290(17):2277-83.

11. Steele RM, Brage S, Corder K, Wareham NJ, Ekelund U. Physical activity, cardiorespiratory fitness, and the metabolic syndrome in youth. J Appl Physiol. 2008;105(1):342-51.

12. Arango CM, Parra DC, Eyler A, Sarmiento O, Mantilla SC, Gomez LF, et al. Walking or bicycling to school and weight status among adolescents from Monteria, Colombia. J Phys Act Health. 2011;8 Suppl 2:S171-7.

13. Wilson PW, D’Agostino RB, Parise H, Sullivan L, Meigs JB. Metabolic syndrome as a precursor of cardiovascular disease and type 2 diabetes mellitus. Circulation. 2005;112(20):3066-72.

14. National High Blood Pressure Education Program Working Group on High Blood Pressure in Children Adolescents. The Fourth Report on the Diagnosis, Evaluation, and Treatment of High Blood Pressure in Children and Adolescents. Pediatrics. 2004;114 Suppl2:555-76.

15. Kuczmarski R, Ogden C, Guo S, Grummer-Strawn L, Flegal K, Mei Z, et al. 2000 CDC Growth Charts for the United States: methods and development. Vital Health Stat. 2002;11(246):1-190.

16. Taylor WC, Baranowski T, Sallis JF. Family determinants of childhood physical activity: A social cognitive model. En: Dishman RK, editor. Advances in Exercise Adherence. Champaign, IL: Human Kinetics; 1994. p. 319-42.
17. Harris TB, Visser M, Everhart J, Cauley J, Tylavsky F, Fuerst T, et al. Waist circumference and sagittal diameter reflect total body fat better than visceral fat in older men and women. The Health, Aging and Body Composition Study. Ann N Y Acad Sci. 2000;904:462-73.

18. McCarthy HD, Ashwell M. A study of central fatness using waist-to-height ratios in UK children and adolescents over two decades supports the simple message-'keep your waist circumference to less than half your height'. Int J Obes (Lond). 2006;30(6):988-92.

19. Hsieh SD, Yoshinaga H, Muto T. Waist-to-height ratio, a simple and practical index for assessing central fat distribution and metabolic risk in Japanese men and women. Int J Obes Relat Metab Disord. 2003;27(5):610-6.

20. Leger LA, Mercier D, Gadoury C, Lambert J. The multistage 20 metre shuttle run test for aerobic fitness. J Sports Sci. 1988;6(2):93-101. Epub 1988/01/01.

21. Van Mechelen W, Hlobil H, Kemper HC. Validation of two running tests as estimates of maximal aerobic power in children. Eur J Appl Physiol Occup Physiol. 1986;55(5):503-6.

22. Liu NY, Plowman SA, Looney MA. The reliability and validity of the 20-meter shuttle test in American students 12 to 15 years old. Res Q Exerc Sport. 1992;63(4):360-5.

23. Welk GJ, Laurson KR, Eisenmann JC, Cureton KJ. Development of youth aerobic-capacity standards using receiver operating characteristic curves. Am J Prev Med. 2011;41 4 Suppl 2: S111-6.

24. Organización Mundial de la Salud. Encuesta Mundial de Salud en Escolares (GSHS). 2012 [2012] [consultado 12 Dic 2013]. Disponible en: http://www.who.int/chp/gshs/es/

25. American Academy of Pediatrics CoPE. Children, adolescents, and television. Pediatrics. 2001;107(2):423-6.

26. Rosner B, Cook NR, Daniels S, Falkner B. Childhood blood pressure trends and risk factors for high blood pressure: The NHANES Experience 1988-2008. Hypertension. 2013;62(2): 247-54.

27. Uscátegui Peñuela RM, Álvarez Uribe MC, Laguado Salinas I, Soler Terranova W, Martínez Maluendas L, Arias Arteaga R, et al. Factores de riesgo cardiovascular en niños de 6 a 18 años de Medellín (Colombia). An Pediatr. 2003;58(5):411-7.

28. Salcedo-Rocha AL, García de Alba JE, Contreras-Marmolejo M. Presión arterial en adolescentes mexicanos: clasificación, factores de riesgo e importancia. Rev Salud Pública. 2010;12(4):612-22.

29. Gomez LF, Parra DC, Lobelo F, Samper B, Moreno J, Jacoby E, et al. Television viewing and its association with overweight in Colombian children: results from the 2005 National Nutrition Survey: a cross sectional study. Int J Behav Nutr Phys Activity. 2007;4:41.

30. Hamilton M, Hamilton D, Zderic T. Role of low energy expenditure and sitting in obesity, metabolic syndrome, type 2 diabetes, and cardiovascular disease. Diabetes. 2007;56(11): 2655-67.

31. Thorp AA, Owen N, Neuhaus M, Dunstan DW. Sedentary behaviors and subsequent health outcomes in adults a systematic review of longitudinal studies, 1996-2011. Am J Prev Med. 2011;41(2):207-15.

32. Borzekowski DL, Robinson TN. The 30-second effect: an experiment revealing the impact of television commercials on food preferences of preschoolers. J Am Diet Assoc. 2001;101(1):42-6.

33. Mallarino C, Gómez LF, González-Zapatall L, Cadenal Y, Parra DC. Advertising of ultra-processed foods and beverages: children as a vulnerable population. Rev Saúde Pública. 2013;47(5):1006-10.

34. Owens J, Maxim R, McGuinn M, Nobile C, Msall M, Alario A. Television-viewing habits and sleep disturbance in school children. Pediatrics. 1999;104(3):e27. Epub 1999/09/02. 
35. Snell EK, Adam EK, Duncan GJ. Sleep and the body mass index and overweight status of children and adolescents. Child Dev. 2007;78(1):309-23.

36. Taheri S. The link between short sleep duration and obesity: we should recommend more sleep to prevent obesity. Arch Dis Childhood. 2006;91(11):881-4. Epub 2006/10/24.

37. Leary SD, Ness AR, Smith GD, Mattocks C, Deere K, Blair SN, et al. Physical activity and blood pressure in childhood: findings from a population-based study. Hypertension. 2008;51(1):92-8.

38. Klasson-Heggebo L, Andersen LB, Wennlof AH, Sardinha LB, Harro M, Froberg K, et al. Graded associations between cardiorespiratory fitness, fatness, and blood pressure in children and adolescents. Br J Sports Med. 2006;40(1):25-9.

39. He Q, Horlick M, Fedun B, Wang J, Pierson RN, Heshka S, et al. Trunk fat and blood pressure in children through puberty. Circulation. 2002;105(9):1093-8.

40. Sorof JM, Lai D, Turner J, Poffenbarger T, Portman RJ. Overweight ethnicity, and the prevalence of hypertension in school-aged children. Pediatrics. 2004;113 3 Pt 1:475-82.

41. Simkin-Silverman LR, Conroy MB, King WC. Treatment of overweight and obesity in primary care practice: Current evidence and future directions. Am J Lifestyle Med. 2008;2:296-304. 